\title{
Case Study: An Unusual Presentation of Smoldering Multiple Myeloma in a patient with End Stage Renal Disease
}

A 59-Year-Old Male patient presented to the benign hematology clinic with a chief complaint of persistent macrocytic anemia with mild thrombocytopenia of unclear etiology. The patient has an extensive medical history including end stage renal disease on hemodialysis post failed kidney transplant and recurrent gastrointestinal bleeds. Two colonoscopies have recently been unable to identify a bleeding source that would account for prolonged anemia. The degree of anemia that the patient was experiencing was disproportionate to clinical documentation of bleeding, and high MCV with normal TSH, Folate, B12 and no alcohol intake ruled out a deficiency as cause of anemia. Iron studies indicated that the cause was Anemia of Chronic disease. A Bone Marrow Biopsy was requested to rule out a Hematologic Malignancy. The Bone Marrow Biopsy came back with a reported diagnosis of Plasma Cell Myeloma as the core biopsy was fifty percent cellular and plasma cells made up fifteen to twenty percent of core biopsy cellularity. Paraprotein and radiologic studies were ordered to further differentiate between multiple myeloma, plasma cell leukemia and smoldering multiple myeloma. Radiologic studies were negative for pathogenic lesions, while paraprotein studies showed a monoclonal gammopathy of IgG Kappa and a bioclonal $\operatorname{IgA}$ region that couldn't be further quantified. The patient was further refered to the malignant hematology Myeloma team. The patient hasn't returned to make any further appointments with hematology, but has been admitted once for complications related to his end stage renal disease. Asymptomatic myeloma, also known as smoldering myeloma, is characterized by having a clonal plasma cell population in the bone marrow that is greater than 10 percent while having no organ damage. Some patients will start out asymptomatic before progressing to full blown disease. Treatment normally begins after symptoms evolve. 